\title{
Robot Path Planning Based on an Improved Multi-Objective PSO Method
}

\author{
$\mathrm{Li} \mathrm{Di}^{1, \mathrm{a}}$, Zheng Zheng ${ }^{1, \mathrm{~b}}$, Meng $\mathrm{Xia}^{2, \mathrm{c}}$ and Min $\mathrm{Xia}^{2, \mathrm{~d}}$ \\ ${ }^{1}$ Economics and Technology Research Institute of State Grid Henan Electric Power Company, \\ Zheng Zhou 450052, China; \\ 2Jiangsu Collaborative Innovation Center on Atmospheric Environment and Equipment \\ Technology, Nanjing University of Information Science and Technology, Nanjing 210044, China. \\ a137886399@qq.com, b1223231819@qq.com, ‘342100300@qq.com, dxiamin@nuist.edu.cn
}

\begin{abstract}
Robot path planning is an important content in the field of robot research. Robot Path Planning is a typical multi-objective optimization problem. The path length, the degree of path smoothness and the degree of security are the optimization objectives in this work. And an improved multi-objective PSO method is used for optimization. In this method, in order to make the particle population multi-objective particle swarm optimization algorithm can quickly converge to the Pareto optimal boundary, an environment selection and a matching selection strategy are proposed. At each iteration of the algorithm, in order to improve the population information exchange and reduce the randomness, the environmental selection and matching selection strategy of SPEA2 are used for multi-objective PSO method, and the particle population can faster convergence to the Pareto optimal boundary. The simulation results verify the method, and the result of proposed method is better than that of multi-objective PSO method, and the simulations indicates that the proposed model is practical for robot path planning.
\end{abstract}

Keywords: Multi-objective evolutionary algorithm; SPEA2; Robot path planning; PSO.

\section{Introduction}

Robot path planning is an important content in the field of robot research [1,2]. This path must satisfy the given performance index, then the robot can safely and without collision to bypass all the obstacles. That the robot path planning is a multi-objective optimization problem [3]. Particle swarm optimization algorithm is a computer technology based on swarm intelligence method [4]. Comparing with other evolutionary algorithms, particle swarm optimization algorithm has an advantage over others is that it has simple, easy and profound biology background, and has fewer parameters need adjusting, which are of great value in use. For particle swarm algorithm, through a lot of single objective optimization problem in research, the result has proved that comparing with other evolutionary algorithms, such as GA, the particle swarm algorithm has faster convergence speed, fewer parameter settings, more simple mathematics description, etc. With many more evolutionary algorithms in multi-objective optimization problems have started using applications such as NSGA2 and SPEA2,etc, then to make further research on multi-objective particle swarm algorithm. However, for multi-objective optimization problem, because of its complexity, it is hard to confirm the more clear optimal position in individual history Pbest and the optimal position in the whole situation gbest.

In standard multi-objective particle swarm algorithm, the choices of the optimal position in individual history Pbest and the optimal position in the whole situation gbest are very random, and the efforts of information exchange between each particle populations are very weak, which have difficulty in let ting the population particles converge to Pareto optimal boundary. On account of those problems in standard multi-objective particle swarm algorithms, so many specialists devote themselves into the research of multi-objective particle swarm algorithms. that there are many scientific achievements., if we use niche technology in multi-objective particle swarm algorithms, adopting Kent mapping, using optimization mechanism, along with searching strategy, bringing in crossover and variation in genetic algorithms, dynamic inertia weight, weeds cloning, etc. $[5,6]$

According to many references [7-10], this paper shows that in standard multi-objective particle swarm optimization algorithms, the environmental selection and pair selection strategy and the calculation method of fitness value in SPEA2 are used [11-13], which are regarded as a evaluation 
standard of multi-objective particle swarm algorithm, then could decrease many stochastic problems of algorithms, and increase the efforts of information exchange between each particle populations so that pick up the speed of population particles converge to Pareto optimal boundary. Through the simulation test of a series of robot path planning, the result has shown that the environmental selection and pair selection strategy and the calculation method of fitness value in SPEA2 could make population particles converge to Pareto optimal boundary quickly, verifying the feasibility and availability of the improved algorithm.

\section{Improved multi-objective particle swarm algorithm}

The processes and procedures of the standard multi-objective particle swarm algorithm :

Step 1: initialization $\mathrm{t}=0$; coordinates of initial search point, which is the initial position $X$ and speed $V$ of the swarm, and non-dominated particle will be added into external archive called nondominated set NDSET .

Step 2: Initialize the optimal position in individual history of each particle populations ,then make pbest $=X$ and the optimal position in the whole situation.

Step 3: under the circumstances of guaranteeing the particle could fly in the searching space, updating the speed and position of each particle, and produce new swarm and the optimal position in individual history.

Step 4: according to new non-dominated solution and pre-existing external swarm to prevent external swarm from spilling. Selecting the optimal position in the whole situation of each particle at the same time.

Step 5: If satisfy the terminal condition, then stop iterating, otherwise transfer $t=t+1$ to step 3 .

Aiming at some disadvantages of standard multi-objective particle swarm algorithm, this paper will make some changes as follows, which are the main innovation points of this paper:

First, for evaluation standard, this paper uses the environmental selection and pair selection strategy and the calculation method of fitness value in SPEA2 to strengthen the efforts of information exchange between each particle populations. On the basic of that to endow fitness values of each particle swarm, which is mean to provide comparison of swarm with a evaluation standard.

Second, for the optimal position in individual history Pbest, when we choose the optimal position in individual history Pbest, this paper will

Select based on pair selection strategy in SPEA2 . Specific operations are shown below:

(1) Former generation swarm unites with latter generation swarm to a new swarm, and give fitness values to each particle, so each particle will has two fitness values: former generation and latter generation.

(2) When we choose the optimal position in individual history, if there has dominance relation between two generation particles, then choose non-dominated individual as the optimal position in individual history of the next generation. If dominance relation does not exist, then choose according to 2 fitness values.

(3) For the optimal position in the whole situation gbest, when we choose it ,it will be selected on the basic of fitness values of each particle to use roulette to choose the optimal position in the whole situation of each particle, rather than random selections.

\section{The evaluation criterion for multi-objective optimization}

How to evaluate the performance of optimization algorithms has been a difficult multi-objective optimization studies, for this, Deb proposed a closer evaluation method. This method is used to calculate the solution set to the reference set or the Pareto optimal solution set minimum distance approach to Measure the extent of algorithm approaching [14]. The smaller of the distance, indicating that the higher approach of the solution set.

The method requires the use of reference sets $P^{*}$ in convergence performance evaluation of a multi objective evolutionary algorithm. The reference set $P^{*}$ is either the Pareto optimal solution set what 
is known, or the non dominated set the non dominated set union. That is $P^{*}=$ nondo minated $\left(\mathrm{U}_{t=0}^{T} \mathrm{NDSet}^{(t)}\right)$, where $\mathrm{NDSet}^{(t)}$ is the non - dominated set of $t$ generation evolution $P^{(\mathrm{t})}(\mathrm{t}=0,1, \cdots, \mathrm{T})$. Because the Pareto optimal solution set of multi - objective problem is generally difficult to obtain, so the reference set $P^{*}$ is usually the non dominated set the non dominated set union. The specific steps are as follows:

First, Calculation of the shortest distance from the non dominated individuals $i$ to $P^{*}$. As the formula (4) is shown below:

$$
P d_{i}=\min _{j=1}^{\left|\mathbf{P}^{*}\right|} \sqrt{\sum_{k=1}^{m}\left(\frac{f_{k}(\mathrm{i})-f_{k}(\mathrm{j})}{f_{k}^{\max }-f_{k}^{\min }}\right)^{2}}
$$

In the formula (4), $f_{k}^{\max }$ and $f_{k}^{\text {min }}$ are the maximum and minimum values of $k$ target in reference set $P^{*}, m$ is the number of sub-objective function.

Then, calculate the average value of a, As the formula (5) is shown below:

$$
C\left(P^{(\mathrm{t})}\right)=\sum_{i=1}^{N D S e^{(t)}} p d_{i} /\left|\mathrm{NDSet}^{(\mathrm{t})}\right|
$$

In order to meet the $C\left(P^{(\mathrm{t})}\right) \in[0,1]$ Do as the formula (6) for processing method is shown:

$$
\bar{C}\left(P^{(\mathrm{t})}\right)=C\left(P^{(\mathrm{t})}\right) / C\left(P^{(0)}\right)
$$

$C\left(P^{(t)}\right)$ is a measure of the multi-objective problem solving set approach degree of value, The smaller the value, shows that the more tends for the solution set to Pareto optimal boundary. Conversely higher the value, the lower tends for the solution set to Pareto optimal boundary. $\bar{C}\left(P^{(\mathrm{t})}\right)$ values between $0-1$, When used to express the multi-objective algorithm convergence speed, The smaller the value, the faster the convergence shows that the solution set, and the greater its value, it indicates that the slower convergence of the solution set

\section{Robot path planning using proposed method}

In the field of intelligent robots, robot path planning is one of the most basic research. Robot path planning involves a lot of problems that we can be reduced to a simple three aspects.

The first is to ensure that the robot does not collide with an obstacle occurs. Therefore, the robot walking path requires a safe distance.

Secondly, the smoothness of the robot path planning is effective.

Finally, there is the impact the robot path length.

We know that the path length, smoothness, the safety distance is not relevant. They satisfy the principle of multi-objective, so we can replace them with three objective functions, it is also seeking optimization of these three functions.

Robot path consists of consecutive line segments $P=\left[s, p_{1}, p_{2}, \ldots, p_{n}, e\right]$, where, $s$ and $e$ represents the start and end points. Mathematical model of multi-objective optimization problem of robot path [15], as follows:

$\min f(P)=\left(f_{1}(P), f_{2}(P), \ldots, f_{n}(P)\right)$

We selected three typical target to optimize and simplify the following function:

$\min F(x)=\left(f_{1}(x), f_{2}(x), f_{3}(x)\right)$

$$
\begin{aligned}
& f_{1}(x)=\sum_{i=1}^{n-1}\left|p_{i} p_{i+1}\right| \\
& f_{2}(x)=\frac{\sum_{i=1}^{n}\left(180-\theta_{i}\right)}{n} \\
& f_{3}(x)=1 / d
\end{aligned}
$$


The above functions on behalf of path length, the average of supplementary angle of two adjacent paths, the reciprocal of safety distance.

In order to get better optimization results, we joined the operator delete route points for robot path problem. If the connecting of $P_{i}$ and $P_{i+2}$ does not intersect with the obstacle, then delete $P_{i+1}$ and Connect $P_{i} P_{i+2}$. Specific operation shown in Figure 1.

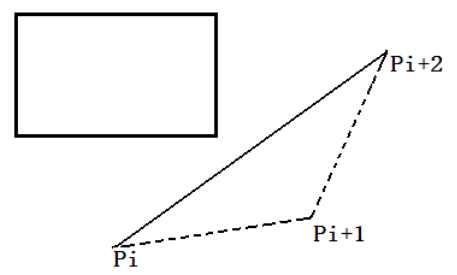

Figure 1 Delete operator operation

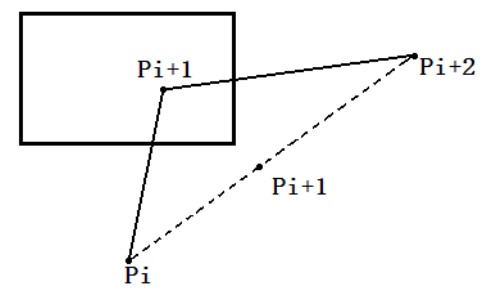

Figure 2 Smoothing operator operation

The smoothing operator is used to remove redundant path points. Smoothing operator can effectively reduce the length of the path of the robot. If the line connect point $P_{i}$ and the point $P_{i+2}$ don't intersect the obstacles, then delete the path point $P_{i+1}$, and connect the point $P_{i+2}$ and $P_{i}$ directly. The operation of the smoothing operator is shown as Figure 2.

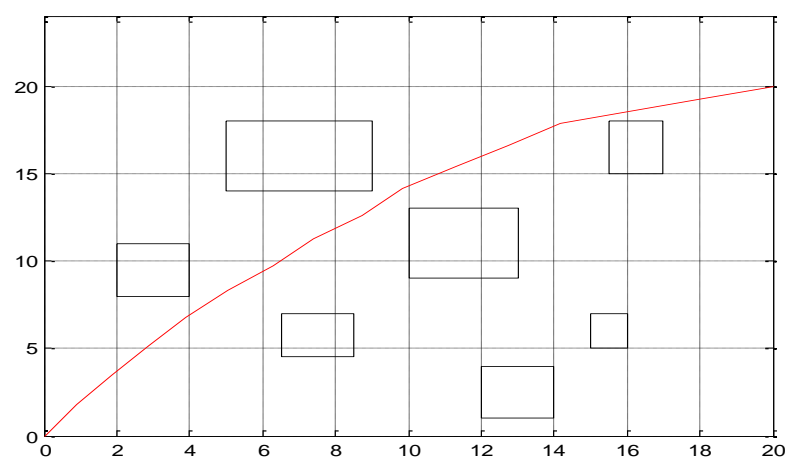

Figure 3 The optimal path of Scene one

In this work, different scenarios are used for the robot path planning, testing the effectiveness of the improved algorithm in solving the robot path planning problem. Figure 3 and Figure 5 are two examples of robot path planning. In the Figure 9 and the Figure 10, the box represents the obstacle, the algorithm needs to find the optimal path between $(0,0)$ and $(20,20)$.

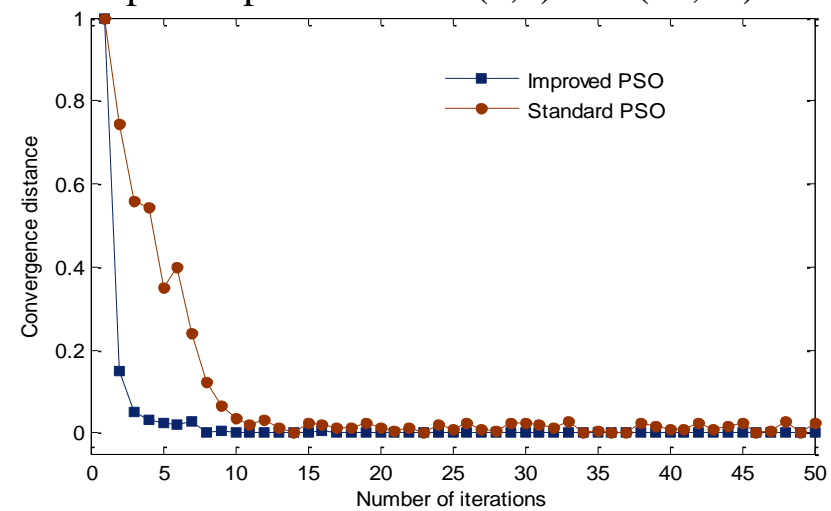

Figure 4 The algorithm convergence of Scene one 


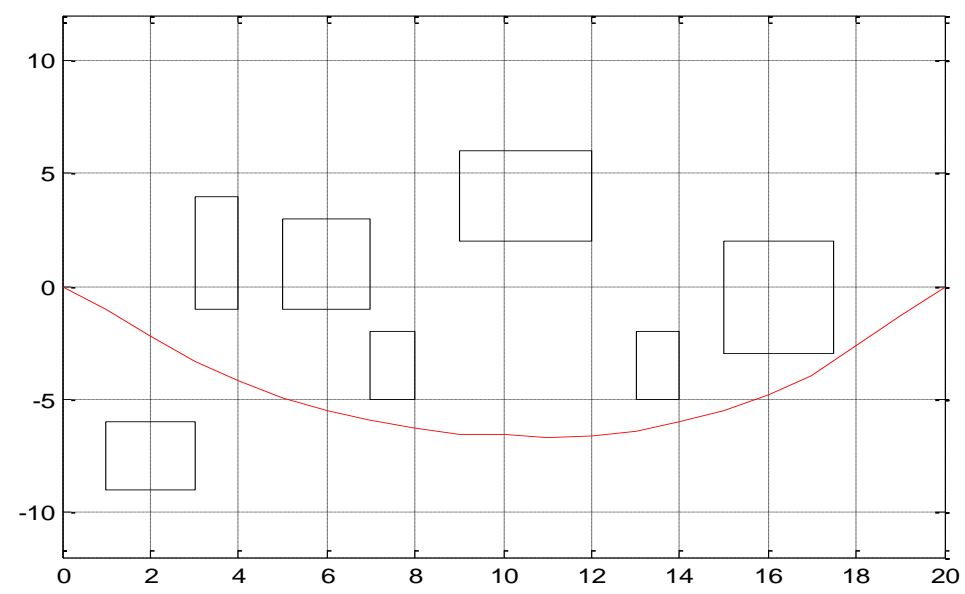

Figure 5 The optimal path of Scene two

From Figures 3 and 5, we can see the length of robot path are the shortest route under the scene, which ensures that the robot with minimal fuel to reach the destination. Relatively smooth path, there is no sharp corners, and the robot always maintain adequate safe distance from obstacles, to ensure that the robot does not collide with the obstacle. Figure 4 and Figure 6 show the convergence of different algorithm for Figure 3 and Figure 5 respectively. In this simulation, the proposed improved PSO method is compared with standard PSO method. Figure 4 and Figure 6 show that the traditional PSO method in the process of convergence appears a lot of shock, and need more iterations to converge to the neighbor of the Pareto optimal set. And the proposed method is also better than traditional PSO method.

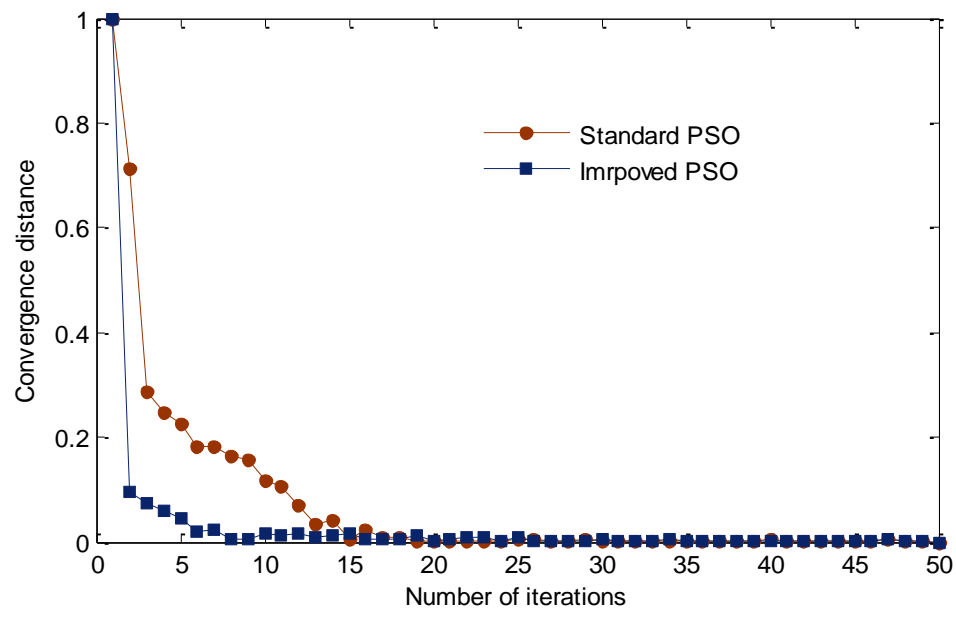

Figure 6 The Algorithm convergence of Scene two

\section{Conclusion}

Robot path optimization is a research hotspot in the field of autonomous robot research. In practical applications, the path optimization is a typical multi-objective optimization problem. In this work, path length, degree of path smoothness, and degree of security are used for optimization. And an improved PSO method is used to solve this problem. The improved algorithm provides an evaluation standard for the improved particle population, in the selection of the best individual position and the global optimal value, the random selection is reduced in multi-objective particle swarm algorithm. And the improved algorithm makes the information exchange among populations strengthened, and the information exchange between each other makes the population more quickly converge to the Pareto optimal boundary. The simulation results show that the improved PSO algorithm convergence ability has been greatly improved, and all the robots walking paths are ideal. It was found that the proposed model is practical for robot path planning. 


\section{Acknowledgments}

This work is supported in part by, supported by National Natural Science Foundation of China (61503192), Six Talent Peaks Program of Jiangsu Province (2014-XXRJ- 007), Perspective Research Foundation of Production Study and Research Alliance of Jiangsu Province (BY2015007-01) and Natural Science Foundation of Jiangsu Province (BK20161533).

\section{References}

[1]. F. Ahmed, K. Deb, Multi-objective optimal path planning using elitist non-dominated sorting genetic algorithms, Soft Computing 17 (2013) 1283-1299.

[2]. M.E. Beniakar, P.E. Kakosimos, A.G. Kladas, Strength Pareto Evolutionary Optimization of an In-Wheel PM Motor With Unequal Teeth for Electric Traction, IEEE Transactions on Magnetics 51(2015)1-4.

[3]. G. Beruvides, Quiza, Ramon, R.E. Haber, Multi-objective optimization based on an improved cross entropy method. A case study of a micro-scale manufacturing process, Information Sciences 334 (2015)161-173.

[4]. P.K. Das, H.S. Behera, S. Das, et al., A hybrid improved PSO-DV algorithm for multi-robot path planning in a clutter environment, Neurocomputing 207 (2016)735-753.

[5]. P.K. Das, H.S. Behera, B.K. Panigrahi, A hybridization of an improved particle swarm optimization and gravitational search algorithm for multi-robot path planning, Swarm and Evolutionary Computation 28 (2016)14-28.

[6]. R. Kala, Multi-robot path planning using co-evolutionary genetic programming, Expert Systems with Applications 39 (2012)3817-3831.

[7]. J. Lee, B.Y. Kang, D.W. Kim, Fast genetic algorithm for robot path planning. Electronics Letters 49 (2013)1449-1451.

[8]. J. Lee, D.W. Kim, An Effective Initialization Method for Genetic Algorithm-based Robot Path Planning using a Directed Acyclic Graph, Information Sciences 332 (2016)1-18.

[9]. H. Li, Q. Zhang, Multi-objective optimization problems with complicated Pareto sets, MOEA/D and NSGA-II, IEEE Transactions on Evolutionary Computation 13 (2009)284-302.

[10]. Y. Ma, H. Wang, Y. Xie, et al., Path planning for multiple mobile robots under doublewarehouse, Information Sciences 278 (2014)357-379.

[11]. D. Martin, A. Rosete, J. Alcal-Fdez, et al., QAR-CIP-NSGA-II: A new multi-objective evolutionary algorithm to mine quantitative association rules, Information Sciences 258 (2014) $1-28$.

[12]. P. Mobadersany, A fuzzy multi-stage path-planning method for a robot in a dynamic environment with unknown moving obstacles, Robotica 33 (2014): 1-17.

[13]. H. Mo, L. Xu, Research of biogeography particle swarm optimization for robot path planning, Neurocomputing 148 (2015) 91-99.

[14]. O. Montiel, U. Orozco-Rosas, R. Seplveda, Path planning for mobile robots using Bacterial Potential Field for avoiding static and dynamic obstacles, Expert Systems with Applications 42 (2015) 5177-5191.

[15]. A. Mukhopadhyay, U. Maulik, S. Bandyopadhyay, et al., Survey of Multi - objective Evolutionary Algorithms for Data Mining: Part II, IEEE Transactions on Evolutionary Computation 18 (2014) 20. 